\title{
Preparing Graduates for the Professions: Achieving Employability through the Exploration of Near-world Scenarios
}

\author{
Edward Peter Errington, James Cook University, Queensland, Australia
}

\begin{abstract}
Scenario-based learning (SBL), founded on situated learning theory and the valuing of contextual knowledge, may provide one stratagem for getting students closer to the realities of their intended profession via the construction and deconstruction of near world scenario experiences in university settings. Scenario-based learning processes present students with realistic sets of circumstances, true-to-life professional tasks, authentic challenges and work-oriented role engagementtransacted through communication styles and within cultural parameters similar to those found in the actual setting. The literature suggests there are four main kinds of near-world scenarios used to enhance the prospects of employability in the professions; namely skills-based, problem-based, issues-based, and speculative-based scenarios respectively. Although each is designed to achieve particular purposes, the author's experience as an academic development adviser in five universities (three countries), suggests that scenario approaches may be chosen arbitrarily, or habitually, by teachers selecting options from a limited knowledge of those available. Given the above, this paper focuses on two fundamental questions: Which SBL features are likely to promote graduate employability? Secondly, how might specific scenario-based approaches embed particular graduate attributes/skills?
\end{abstract}

Keywords: Higher Education, Scenario-based Learning, Employability, Graduate Preparation

\section{Introduction}

$\mathrm{A}$

N EXPANDING STUDENT population, widening participation and the subsequent pressure on work placement opportunities where students, as would-be professionals can articulate their experience has resulted in a re-evaluation of what is possible in preparing graduates for the professions.

A review of the literature suggests that scenario-based learning (SBL), founded on the principles of situated cognition and the valuing of contextual knowledge is one stratagem that might be used to optimise work experience and prepare graduates for the professions by providing rich opportunities for students, as would-be professionals to gain contextual knowledge and, by so doing, become more "future-proofed" (Hill, Popovic, Eland, Lawton and Morton, 2010,p.27).

Naidu (2010) observes that both scenario-based learning (SBL) and work-based professional practice focus on 'authentic' learning tasks and assessment, "and as such SBL has the potential to offer graduating students the requisite skills and competencies for entering the workforce", and "the apprenticeship that graduating students need", (p.47).

It is not suggested that scenario-based learning should substitute work-based placements, but rather provide an appropriate vehicle for supplementing lived professional experience. Experience shows that educators for the professions often draw their scenario content, dilem-

The International Journal of Interdisciplinary Social Sciences

Volume 5, Number 5, 2010, http://www.SocialSciences-Journal.com, ISSN 1833-1882

(C) Common Ground, Edward Peter Errington, All Rights Reserved, Permissions:

cg-support@commongroundpublishing.com 
mas and tasks directly from the work-based experiences of their students in re-creating events for experiential and reflective purposes.

Clearly the potential is there for scenario-based learning to provide ways of meeting the demand for relevant professional preparation if planned, delivered, evaluated, and reflected upon in a systematic way to achieve graduate employability.

Given the above, this paper focuses on two fundamental questions: Which SBL features are likely to promote graduate employability? Secondly, how might specific scenario-based approaches embed particular graduate attributes/skills? The first task, however, is to define 'scenario-based learning' and its inherent processes.

\section{Defining Scenario-based Learning}

The term 'scenario-based learning' refers to any educational approach that involves the use or dependence upon, scenarios to bring about desired learning intentions. Scenarios may constitute a given set of circumstances, a description of human behaviour, an outline of events, a story of human endeavour, an incident within a professional setting, or human dilemma, (Errington, 2003,p.12). The common thread is in using scenarios to achieve specific educational purposes. A review of the literature reveals a plethora of names for SBL. Common to all is the notion that the scenario or situation carries the learning experience.

In this paper, scenarios are deemed 'near-life' rather than 'real-life' in acknowledging that simulations can never be the real-world. The real-world is just so: scenarios are mental fabrications that can only replicate aspects of life - rather like snapshot images. The fact that these snapshots can be examined in minute detail from a range of perspectives, gives some indication as to their value in helping formulate aspects of 'professional identity'. A scenario can be dissected, re-run many times over, and sited in a different time or place.

In a scenario-based learning process, students as potential professionals are presented with a scenario descriptor (or set of circumstances) and one or more focus questions to pursue lines of inquiry along possible pathways. Students assume specific roles or perspectives that allow an exploration of various scenario vantage points. Through the crafting of the scenario (delivered whole, or incrementally) and the judicious choice of focus questions, students are placed in a position where they can demonstrate a skill/procedure, pursue a problem, explore an issue, and/or speculate on professional knowledge without experiencing the consequences of failure that the real-world can impose, (Aitken, 2010, p.75).

Scenarios are usually scaffolded by discussion, guided observation, role-play and/or debate, interjected by periods of reflection. The choice of "scaffolding" often reflects the cultural sites of the specific profession: For example, law students may engage in a 'courtroom debate' within a moot court, discuss issues as a (quasi) 'legal team', and deliberate on cases as legal colleagues.

\section{Scenario-based Learning Qualities Promoting}

\section{Graduate Employability}

In order to respond to the question: 'Which SBL features are likely to promote graduate employability?' it is necessary to review SBL qualities revealed by the literature. The evidence 
is predominantly anecdotal. Educators cite examples of SBL where it is used to help students, (as would-be professionals) to:-

(i) Bridge the gap between subject theory and the realities of professional practice SBL is used to contextualise theoretical and practical knowledge within professional settings: For example, Adam (2010) observes how and why SBL can provide a bridging strategy between 'top-down' theorising in pre-service teacher education programs and the 'bottom-up' realities of school-based teaching (p.97).

(ii) Provide authentic (professional focused) contexts for learning

The emphasis is placed on providing students with 'authentic learning' situations and 'relevant' tasks as they relate to the reality of current professional settings: For such authentic learning to occur, every effort must be made by educators to situate it within real life experiences. While exploring realistic dilemmas, students are urged to realise, as in real life, there is no one single, correct solution to these enigmas, (Sorin, 2010, p.148).

(iii) Face realistic and relevant situations/contexts

Scenarios can offer the closest experience to 'reality' within the simulated professional setting (Errington, 2009, p.585). Realism may be generated by pursuing genuine problems in a realistic way, that is, problems are based on 'genuine' issues (where outcomes have not been predetermined). It is clearly important that educators keep abreast of the profession's current profile by maintaining close partnerships with employers and professional bodies and valuing students' experiences encountered on work placements. (iv) Introduce students to the culture of the workplace

Lave and Wenger, (1991) note that "being able to speak the vocabulary and tell the stories of a culture of practice is fundamental to learning" (quoted in Herrington and Oliver, 1995, p.6). Scenarios may provide snapshots of the culture - partial 'stories' of the profession's past, present and possible future. Members of the culture constitute 'communities of practice', (Hebron in Kindley, 2002, p.3), wherein students acquaint themselves with the norms and practices of the profession to survive and prosper. The scenario has the potential to carry the technical and informal language, etiquette, roles, challenges, tasks, problems, relationships, key responsibilities, ethics and values of the profession, (Errington 2009, p.585). This potential for immersion in the culture is far removed from a theory-only view of the professions espoused by more traditional professional courses.

(v) Analyse experience to make appropriate professional interventions

Hill et al. (2010) note that "students graduating as practitioners in any field must be capable of recognising and analysing situations to identify when an intervention is required, and be able to formulate potential options from which they can rationalise the selected course of action", (p.29). These professional skills are developed, practised and reinforced by specific scenarios.

(vi) Contemplate and develop a professional identity

The duel concept of student as learner and would-be professional is an important one for graduate employability: Connecting both are notions of 'personal and professional identity'. It would appear crucial that aspiring professionals are able to envision and explore alternative futures in order to develop the kind of flexibility needed to negotiate events and issues from a professional/ multi- perspective. Participation in an SBL process 
would arguably constitute identity construction - not only in terms of who these aspiring students are now as potential lawyers or nurses, but also in respect of what kind of lawyer or nurse they aspire to be. Scenarios can facilitate an interrogation of 'identity' with its attendant roles, responsibilities and 'professional thinking'.

An important feature of SBL is the opportunity it affords for critical reflection on experience in formulating a professional identity: For example, Miller and NambiarGreenwood (2010) employ scenarios to unravel students' 'live' clinical problems and 'life' issues experienced on their professional journey, scaffolded by opportunities for reflection in and on individual/shared experience (p.111).

(vii)Practise professional teamwork/collaboration

Successful scenario-based learning operates within positive social frameworks so that meaningful (task/goal-driven) collaborations sited in professional settings become possible. For teams to work successfully, it is important that teachers and students maintain an atmosphere conducive to learning; one in which students do not feel threatened or exposed, and feel able to proffer alternative opinions and solutions (Errington, 2005, p.67).

(viii) Develop cultural awareness through transformed practice

Ireland (2010) asserts that the main challenge of education for the professions is to create a learning environment which fosters learner transformation where 'situated practice' becomes 'transformed practice' in transforming the cultural awareness of potential graduates. She uses scenarios to engage students in transformative processes. Hill et al. note that cultural awareness and client sensitivity are two skills often ignored in university teaching, and "such skills are often critical to the development of the selfawareness new graduates need as they enter the workplace" (p.27).

In summary, SBL characteristics are associated with graduate employability in terms of bridging subject theory and professional practice; providing a realism that reflects the professional work space and its inhabitants; introducing the culture of the profession; creating opportunities to analyse and reflect on experience and formulate appropriate interventions; facilitating the formation of professional identity; creating teamwork/collaborative and interpersonal opportunities similar to those found in the workplace; and, constructing a vehicle for transformative cultural awareness.

It is reasonable to assume SBL is likely to have most impact on graduate employability prospects if and where specific approaches are chosen to target particular graduate attributes: 'How might specific scenario-based approaches be used to embed particular graduate attributes associated with employability?' What kinds of scenario options are available to educators, and what is their potential 'goodness of fit' with the graduate employability attributes found in the mission statements of UK and Australian universities?

\section{Achieving Graduate Employability through Specific Kinds of Scenario Approaches}

Surveys carried out in 2003, 2005 and 2009 suggest four main kinds of scenarios are used by university teachers to instil specific graduate attributes within/across a range of professional disciplines: These are skills-based; problem-based; issues-based; and speculative- 
based scenarios respectively. Together these main options incorporate many of the scenario offerings used to promote graduate employability in tertiary education.

What follows is a discussion on how and why each respective approach might add to the employability prospects of graduates when chosen judiciously.

\section{- Skills-based scenarios}

Skills-based scenarios provide structures to allow students to demonstrate what they have learned or understand practically and theoretically. For example, trainee nurses demonstrate their clinical skills within simulated hospital settings for assessment (feedback) purposes, (Gammer, 2003; van Wissen, 2003). Elsewhere, information technology students demonstrate their technical knowledge and interpersonal communication skills in a simulated consultantclient setting, (Bell and Page, 2003).

Naidu (2010) notes that "scenarios provide a context to anchor and ground the facts, principles and procedures that need to be learned and taught", (p.44). Using skills-based scenarios, students rehearse and reflect on selected attributes until they are internalised using explicit criteria as a guide for appropriate professional behaviour.

Skills-based scenarios appear most useful when knowledge seems 'fixed', possesses clear steps, or procedures; where abilities lend themselves to practical expression; notions of 'appropriate professional behaviour' are firmly defined; and, role expectations and responsibilities are explicit.

Desirable graduate employability attributes such as teamwork, communication skills, presentation skills, information literacy, and cultural awareness may be practised and embedded using a skills-based approach (Errington, 2003, p.17).

\section{- Problem-based scenarios}

In problem-based approaches, students pursue specific open-ended problems where they are required to identify what they know already about the problem; draw upon working knowledge; locate that knowledge in the discipline area; construct knowledge en-route; apply these gleanings to a series of challenges; react appropriately to problems as they arise; and, arrive at considered solutions based on reasons that can be justified. This specific process is designed to engage students in the pursuit of realistic problem where the process is deemed more valuable than the "final" solution(s) and follows a problem-based learning rationale found in the literature (See for instance, Savin-Baden, 2000).

A common example involves the diagnoses of (real/virtual) patients by trainee medical practitioners. Here is an adapted example:-

\section{'Diagnosis'}

A patient has just been admitted to Ward 4. She speaks little, if any English, refuses to be physically examined, and seems to be complaining about her throat. Her tongue is covered in black fur, and she has vomited twice. What are the underlying problems here? Why?

In the above example, there is no obvious singular problem or 'solution'. Establishing what the problem(s) is must be a first priority. Students may be asked what they know of similar circumstances (possibly from knowledge met in the course to date). What do they need to 
know to fill in missing knowledge gaps? How will they go about finding this information? How will findings/evidence be evaluated by those gathering it? How will it be presented? How will their findings be evaluated by the class/teacher? The process will incorporate moments for deliberation and reflection

Problem-based scenarios are useful for applying knowledge in demanding circumstances/series of challenges; responding spontaneously to changing circumstances; exploring issues and events at a deep level over a lengthy period of time; exploring 'consensus' and 'difference' as part of human dynamics; engaging students in processes of decision-making; and for constructing new knowledge as appropriate (Errington, 2003, p.23). Here, the journey is deemed more important than the destination. Even simple decisions will have consequences, and often students report that so-called 'simple' choices often turn out to be more complicated than originally thought.

In problem-based scenarios, perspective professionals are required to tolerate "messy" ("life-like") problems and appreciate the value of ambiguity and uncertainty, and present ideas to peers for evaluation, critique and reflection. This can be a big challenge for many aspiring professionals (See McLoughlin, Hogg and Darvell, 2003; Miller, Smailes, Stark, Street and Watson, 2003).

Problem-based scenarios appear in a variety of trainee professional contexts, including: For example, in helping students respond to global disasters as trainee disaster educators, (Aitken, 2010); meeting the creative design demands of industry clients (Fleischmann and Daniel, 2010); engaging in a bridging strategy between 'top-down' theorising in pre-service teacher education and the 'bottom-up realities of school-based teaching (Adam, 2010); and, in developing a professional identity in mental health nursing (Miller and Nambiar-Greenwood, 2010).

Graduate attributes developed via problem-based scenarios are likely to include decisionmaking skills, teamwork, higher order reasoning, presentation skills, the ability to gather evidence to support hypotheses, critical thinking, tolerance of ambiguity, ability to evaluate and disseminate complex information, and self-awareness among others.

\section{- Issues-based scenarios}

Issues-based approaches employ scenarios to promote potential graduates' understanding of a range of contextual issues and concerns underpinning and/or informing a profession.

Participants are required to investigate the attitudes, beliefs and values surrounding issues; seek out the beliefs, attitudes held by the relevant human agencies; take a particular stand/role/perspective on an issue; enter a scenario situation where this stand is revealed, articulated, possibly defended and evaluated in relation to similar and dissimilar positions represented by other students; negotiate or contend with those holding dissimilar positions; and, possibly, take an opposing stand on an issue. One aim of this approach is to enable students to contrast and compare positions taken - alongside the development of empathy for the views of others. Role-takers may be brought together to debate the issue at a simulated 'public' meeting. Alternatively, student engagement may remain at the level of discussion or enter into deeper level role-play, (Errington, 2005, p.24).

Issues-based scenarios can provide future graduates with an opportunity to articulate professional issues with confidence supported by substantive evidence. It is important for potential professionals to become shrewd observers of human behaviour, noting the impact 
of vested interests on all aspects of their profession. Much may be gained by spending time in 'other people's shoes', particularly those of prospective clients, patients and dependent others.

Issues-based scenarios appear popular among educators in the social sciences. For example, Holm (2010) observes the ways that potential legal educators use these scenarios to identify relevant legal issues, apply the relevant legal concepts, employ legal reasoning and proffer advice about the issues and the scenario approach itself (p.169). Elsewhere, Nickson (2010) describes the ways that issues-based scenarios are used in social work and community welfare practice to make ethical decision making more relevant to students (p.211).

Graduate attributes developed via issues-based scenarios are likely to include the ability to recognise multiple perspectives on any 'singular' issue, decision-making skills, teamwork, higher order reasoning, presentation skills, the ability to gather evidence to support hypotheses, critical thinking, evaluating of information, and self-awareness among others.

\section{Speculative-based Scenarios}

Within a speculative scenario format, students are given, or seek out current knowledge about the profession (data, facts, information, trends, social actors), and either postulate on aspects of the future, as it might become, or on aspects of the past as these might have happened. Scenarios are used to generate ideas, formulate and test hypotheses, and support deliberations with evidence. Participants in the (speculative) planning process generate their own/shared perceptions of the future of the profession. These futures are not so much based on notions of probability, but rather on 'degrees of fit' with the contemporary realities of what students believe, and do know already about the present, (Errington, 2005, p.25). Students realise that what is said and done now has implications for everyone as all knowledge is seen to be related.

The past and future can be viewed as 'problematic' with few fixed points. Students are prompted to speculate on the uncertainties surrounding knowledge and held beliefs; to facilitate legitimate expression of their own/other's attitudes and feelings; and, to develop empathetic understanding. Though close to both problem-based and issues-based scenarios, a speculative example will incorporate elements of time (past, present, future) and space ('taking' students from one place to another), demanding from students sophisticated projection, imagination and intuition, as well as 'the facts'.

Two perennial questions are used to interrogate speculative-based scenarios: 'What would happen if?' (future-oriented scenario, eg. potential of company success in business studies) and, 'Why did this happen in this way?' (past-oriented scenarios, eg. used in forensic science to re-create past events).

Murray (2010) provides an example of a speculative scenario she offers to combined cohorts of sociology and veterinary science students. Students assume roles as extra- terrestrial sentient beings from a planet in another galaxy. They consider reasons why they, as sentient beings from outer space, might place members of the human species into zoos. The scenario is used to explore problematical ethical issues surrounding the practice of keeping non-human animals in zoos, offering an imaginative and lively engagement with human, animal, and environmental rights and concerns. It acts as a metaphor for all 'zoos', or places of confinement, and the issues raised by having such places. Students as aspiring sociologists or 
veterinary scientists are required to think holistically around a collection of problems and issues underpinning the scenario (p.223).

Graduate attributes developed via speculative-based approaches are likely to include decision-making, teamwork, critical thinking, higher order reasoning, presentation skills, the ability to gather evidence to support hypotheses, predictive skills, data evaluation, trend analysis, creativity and evaluation of information. Students as future professionals deliberate on past and/or future issues and problems based on a blend of current working knowledge, acquired professional knowledge, tacit knowledge, intuition, and creativity, supported by (current) evidence/artefacts.

All four approaches may be used singularly, or in combination to contribute towards specific graduate attributes. There is potential for introducing one or more skills-based, problem-based, issues-based and speculative-based scenarios at different staging points in the formation of professional identity.

\section{Conclusion}

The paper began by identifying some general qualities or properties of scenario-based learning approaches that lend them to the promotion of graduate employability via the judicious use of scenarios in response to the question: 'Which scenario-based learning qualities might be harnessed to promote graduate employability?' By so doing, the paper highlighted some of the main constituents of scenario-based learning processes in relation to how and why such processes might optimise graduate employability in the professions.

From here, the paper outlined the characteristics of specific kinds of scenario approaches and the graduate attributes they might embed within the curriculum and subsequent professional practice, in response to the question: 'How might specific scenario-based approaches embed particular graduate attributes associated with employability?' There are clear differences between approaches - enough to warrant their judicious choice by educators for the professions.

Overall, SBL has a number of significant features that may be harnessed to supplement real work-based experience and accord students a degree of future-proofing through the fostering of adaptability to change and a tolerance of ambiguity and uncertainty found in all professions. Practical endeavours are likely to prove more successful when specific kinds of scenarios are aligned with specific graduate attributes.

\section{References}

Adam, R. (2010). Schooling for hard knocks: Using scenario-based learning (SBL) for behaviour management skills in pre-service teacher education. In E.P.Errington (Ed.), Preparing graduates for the professions using scenario-based learning. (pp 97-109). Brisbane, Australia: Post Pressed.

Aitken, P. (2010). Scenario-based Disaster Health Education: "War stories" asvicarious experience. In E.P. Errington (Ed.), Preparing graduates for the professions using scenario-based learning. (pp 75-84). Brisbane, Australia: Post Pressed.

Bell, S. \& Page, R. (2003). Paper to Program: Developing a computer-based learningpackage for SBL. In E.P.Errington (Ed.) Developing scenario-based learning: Practical insights for tertiary teachers. (pp50-61). Palmerston North, New Zealand: Dunmore Press.

Errington, E. (Ed.) (2010). Preparing graduates for the professions using scenario- based learning. Brisbane, Australia: Post Pressed. 
Errington, E. (2009). Being There: Closing the gap between learners and contextualknowledge using near-world scenarios. The International Journal of Learning, 16 , (8 ), pp. 585-594.

Errington, E. (2005). Creating Learning Scenarios: A planning guide for adult educators. Palmerston North, New Zealand: Cool Books.

Errington, E. (2003). (Ed.) Developing scenario-based learning: Practical insights for tertiary teachers. Palmerston North, New Zealand: Dunmore Press.

Fleischmann, K. \& Daniel, R. (2010). Enhancing employability through the use ofreal-life scenarios in digital design education. In E.P. Errington (Ed.), Preparing graduates for the professions using scenario-based learning. (pp85-96).Brisbane, Australia: Post Pressed.

Gammer, S. (2003). Demonstrating professional skills through scenario-basedlearning. In E.P.Errington (Ed.) Developing scenario-based learning: Practical insights for tertiary teachers. (31-39). Palmerston North, New Zealand: Dunmore Press.

Herrington, J. \& Oliver, R. (1995). Critical characteristics of Situated Learning:Implications for the Instructional Design of Multimedia. Ascilite Conference,Melbourne. Retrieved September $1^{\text {st }}, 2009$ from:http://www.ascilite.org.au/conferences/melbourne95/smtu/papers/herrington.pdf

Hill, A., Popovic, C., Eland, J., Lawton, R., \& Morton, N. (2010). Creating future-proof graduates using scenario-based learning. In E.P. Errington (Ed.), Preparing graduates for the professions using scenario-based learning. (pp27-37). Brisbane, Australia: Post Pressed.

Holm, E. (2010). Using real-life scenarios in law to prepare graduates for professionalwork practices. In E.P. Errington (Ed.), Preparing graduates for the professions using scenario-based learning. (pp169-176). Brisbane, Australia: Post Pressed.

Kindley. R. (2002). Scenario-based e-Learning: A Step Beyond Traditional E-Learning. Retrieved July 14, 2010, fromhttp://www.learningcircuits.org/2002/may2002/kindley.html

Lave, J. \& Wenger, E. (1991). Situated learning: Legitimate peripheral participation. Cambridge, UK: Cambridge University Press.

McLoughlin, M., Hogg, C. \& Darvill, A. (2003). Rather than being told: UsingSBL to raise cultural awareness in nurse education. In E.P. Errington(Ed.)Developing Scenario-based learning: Practical insights for tertiary teachers. (pp92-101). Palmerston North, New Zealand: Dunmore Press.

Miller, E. \& Nambiar-Greenwood, G. (2010). Understanding experience: The collaborative journey using scenario-based learning. In E.P. Errington (Ed.), Preparing graduates for the professions using scenario-based learning. (pp111-120). Brisbane, Australia: Post Pressed.

Miller, E., Smailes, S., Stark, S., Street, C. \& Watson, K. (2003). Craving(un)certainty: Using SBL for teaching in health care contexts. In E.P.Errington (Ed.), Developing Scenario-based Learning:Practical insights for tertiary educators. (pp102-112). Palmerston North, New Zealand: Dunmore Press.

Murray, M. (2010). The Human-Animal Zoo: Exploring enclosure, species and space. In E.P. Errington (Ed.), Preparing graduates for the professions using scenario- based learning. (pp223-233). Brisbane, Australia: Post Pressed.

Naidu, S. (2010). Using scenario-based learning to promote situated learning anddevelop professional knowledge. In E.P. Errington (Ed.), Preparing graduates for the professions using scenariobased learning. (pp39-49). Brisbane, Australia: Post Pressed.

Nickson, A. (2010). Social work ethics in scenario-based learning. In E.P. Errington (Ed.), Preparing graduates for the professions using scenario-based learning. (pp211-222). Brisbane, Australia: Post Pressed.

Orey, M.A. \& Nelson, W.A. (1994). Situated Learning and the Limits of Applying theResults of These Data to the Theory of Cognitive Apprenticeships. InM.R.Simonsen (Ed.), Proceedings of Selected Research and Development Presentations at the 1994 National Convention of The Association for Educational Communications and Technology. Washington DC: AECT. [ED 373 746] 
Savin-Baden, M. (2000). Problem-based learning in higher education: Untold stories,Buckingham, UK: Open University Press.

Sorin, R. (2010). Webfolio - 'Real-life' scenarios in an online learning environment.In E.P. Errington (Ed.), Preparing graduates for the professions using scenario- based learning. (pp147-157). Brisbane, Australia: Post Pressed.

Van Wissen, K. (2003). Developing reflective practice through scenario-basedlearning. In E.P.Errington (Ed.) Developing scenario-based learning: Practical insights for tertiary teachers. (pp40-49). Palmerston North, New Zealand: Dunmore Press.

\section{About the Author}

Dr. Edward Peter Errington

Dr. Edward Errington is an academic development adviser based in the Teaching and Learning Development section of James Cook University (JCU). He has a background as a primary \& secondary drama teacher, arts educator, teacher educator, and academic development adviser in the UK, New Zealand and Australia. At JCU, he works with teachers from all disciplines to help them provide authentic and relevant learning opportunities for their students. He has presented scenario- based work in 12 countries so far, and has published six non-fiction books and numerous book chapters and articles on the efficacy of scenariobased learning approaches in higher education. 


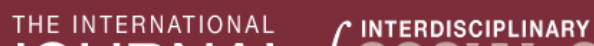 JOURNAL Of SOGHAL SGIENGES}

\section{EDITORS}

Mary Kalantzis, University of Illinois, Urbana-Champaign, USA.

Bill Cope, University of Illinois, Urbana-Champaign, USA.

\section{EDITORIAL ADVISORY BOARD}

Patrick Baert, Cambridge University, Cambridge, UK.

Norma Burgess, Syracuse University, Syracuse, USA.

Bill Cope, University of Illinois, Urbana-Champaign, USA.

Peter Harvey, University of Adelaide, Adelaide, Australia.

Vangelis Intzidis, University of the Aegean, Rhodes, Greece.

Paul James, RMIT University, Melbourne, Australia.

Mary Kalantzis, University of Illinois, Urbana-Champaign, USA.

Gerassimos Kouzelis, University of Athens, Athens, Greece.

Massimo Leone, University of Turin, Turin, Italy.

Alexandros-Andreas Kyrtsis, University of Athens, Athens, Greece.

José Luis Ortega Martín, Universidad de Granada, Granada, Spain.

Bertha Ochieng, University of Bradford, Bradford, UK.

Francisco Fernandez Palomares, Universidad de Granada, Granada, Spain.

Miguel A. Pereyra, Universidad de Granada, Granada, Spain.

Constantine D. Skordoulis, University of Athens, Athens, Greece.

Chad Turnbull, ESADE Business School, Barcelona, Spain.

Chryssi Vitsilakis-Soroniatis, University of the Aegean, Rhodes, Greece.

Please visit the Journal website at http://www.SocialSciences-Journal.com for further information about the Journal or to subscribe. 


\section{THE UNIVERSITY PRESS JOURNALS}

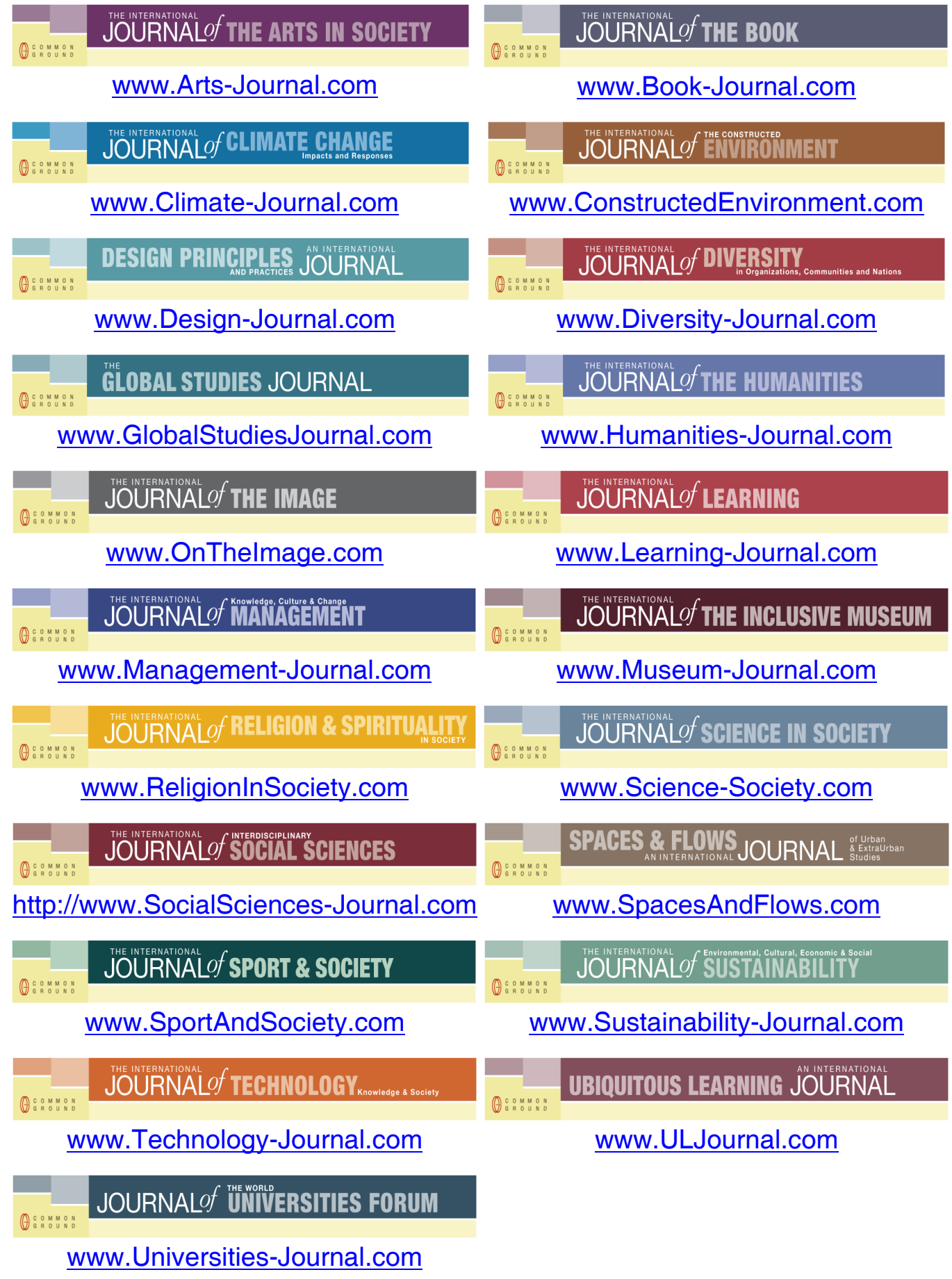

FOR SUBSCRIPTION INFORMATION, PLEASE CONTACT subscriptions@commongroundpublishing.com 